\title{
Second COVID-19 Surge: Challenges and Handling
}

\author{
Rakesh Lodha $^{1}$ · S. K. Kabra ${ }^{1}$ \\ Published online: 5 May 2021 \\ (C) Dr. K C Chaudhuri Foundation 2021
}

Coronavirus disease 2019 (COVID-19) pandemic is ongoing for more than a year and now we are facing the second wave. The sharp jump in the number of cases being reported, and possibly some change in the disease profile have seriously challenged the available infrastructure to breaking point; its adverse impact on the quality of care is being reflected in the outcomes. Once again, the medical fraternity is facing challenges of managing overwhelming emergencies in background of inadequate resources [1]. The situation is very grim with significant shortage of trained manpower, hospital beds (all type, more of ICU beds), drugs, and oxygen. Let us analyze the possible reasons and whether we could have avoided this situation and get ready to face such problems in future.

In the current surge, a common perception is that children are more frequently being affected in second surge and they are relatively sicker, unlike the scenario in 2020 . The data collected in Integrated Disease Surveillance Programme (IDSP) and the Indian Council of Medical Research suggest that the proportion of individuals $0-10 \mathrm{y}$ age and $11-20 \mathrm{y}$ age among the confirmed COVID cases has not changed much since last year [2]. In view of the increase in overall cases, the absolute number of children affected has increased. Overall, the outcomes in children continue to be good [2].

Multiple factors seem to have contributed to the increase in overall numbers of cases as well as cases in children: poor compliance of the population to COVID-appropriate behavior, large gatherings across the country, no strict action to break the spread of infection in households and community, opening up of schools/colleges, and possibly a mutant strain that is more contagious. There was a significant discordance between the messages being given by the authorities and their other actions, allowing/promoting inappropriate behaviors.

S. K. Kabra

skkabra@hotmail.com

1 Department of Pediatrics, All India Institute of Medical Sciences, New Delhi 110029, India
The behavior of the population seems also to have been influenced by the fact that the recovery rate is more than $98 \%$, an underestimation of the potential severity, and an attitude that even if one is diagnosed as COVID-19 infection, he/she will not be isolated in hospitals as happened in the first surge. In addition, the approval of vaccines may have given a misplaced sense of hope, further encouraging lowering of guard.

Before the first COVID-19 surge in India, media and social media played overenthusiastic role, and various experts who did not have adequate knowledge but were media-savvy played an important role in creating hype to make COVID19 equivalent to death. Gradually media started reporting very high recovery rate and low case fatality rates, which improved public confidence, but over a period of time, also led to lowering of guard. Community fatigue setting in along with economic stress, further led to increased risk-taking behaviors in context of COVID-19. A lesson learnt is that role of electronic media cannot be denied but there is a need to take balanced, mature approach keeping in mind the ramifications of actions.

After a few weeks into the pandemic, it had become clear that we needed to learn to live with COVID-19 for months to year, as it is going to stay with us. Going by the records of previous pandemics, a second or third surge was always on the cards. Specifically, in the previous pandemic of Spanish flu in 1918, there was more devastating effect of pandemic during the second surge [3]. A few experts warned of a similar course with COVID-19 as well. However, the response of most countries, including ours, was less than desirable even after Europe and America had seen the surge. Even when the surge started in Maharashtra and Kerala in India, there seemed to be a lack of proactive measures to build capacity and arrange resources to face the surge in other parts of the country. These warning signals were ignored or not given due regard. Response of leadership and policymakers from other parts of India and experts were confined to reassurance, only suggesting that our city/state is fine, the infection rate is much less than the other states [4]. Even large gatherings have been promoted in various parts of the country. Another lesson learnt is that if an 
airborne infection occurs in one part of the country or world, it will not remain confined to a particular region; hence, a proactive action to effectively control at the source and effective measures to stop transmission of infection have to be instituted to contain outbreaks. Regarding predictions, it is imperative to err on side of predictions of higher numbers rather than having wishful thinking.

With the approval of vaccines, more emphasis was given to vaccination, forgetting that for immediate control, there was a need to break transmission of infection, and for long-term control vaccination plays an important role. The vaccines definitely have a role in preventing severe disease. However, to think that vaccination will have an important role in immediate control of COVID-19 in the country, given the limited availability of vaccine stock and time required to cover a significant proportion of population, appears illogical; more so, that the efficacy of vaccines is not too high and these may not prevent a person from getting infected and potentially contributing to the spread of infection. Vaccine hesitancy among the healthcare workers in the beginning also contributed to some delay in vaccination drive; lack of transparency in the approvals of vaccines seems to have contributed too. Similarly, approval to various drugs for COVID-19 in the absence of robust evidence did little to boost the confidence of the fraternity.

We did not use the 'lull period' to prepare ourselves. The window of opportunity of a few months was clearly wasted and the results are very obvious in the form of extreme shortage of hospital beds, oxygen, pharmaceutical agents, trained healthcare staff, etc. Proposals for establishing oxygen plants in various centers appear to have been delayed. It almost amounts to abdication of responsibilities and common man being left on his own to arrange for the basic healthcare.

At the beginning of COVID-19 pandemic, we were ignorant about most of the aspects of this new disease. Now, we have substantial information about the pandemic, and significant experience, knowledge of course of illness and management. From first surge to second surge, we have seen multiple pharmacologic agents, including hydroxychloroquine (HCQ), remdesivir, ritonavir-lopinavir, ivermectin, etc. being suggested as potential therapies. Several randomized, controlled trials and other studies have been carried out to document efficacy of various pharmaceutical agents. The World Health Organization (WHO) has issued living guideline about various therapeutic agents and provided evidence-based guideline for management of COVID-19 infections [5]. Though the WHO has clearly defined that there is no definitive role of any of the pharmaceutical agents for treatment or prophylaxis except therapeutic role of corticosteroids in severe and critically sick patients, medical fraternity continues to use many of the drugs in all severity of COVID-19 resulting in a shortage of drugs. In the first surge, drugs like HCQ disappeared from market and people were buying them at huge prices. Now in the second surge, there is an extreme shortage of tocilizumab, remdesivir, ivermectin, favipiravir, etc. The reasons for not following rational guidelines may be multiple: lack of dissemination of evidence-based information to stakeholders (medical fraternity), desperate use of various agents in absence of proven therapies, or significant heterogeneity in studies due to variation in methods. Additionally, some guidelines suggest use of antivirals [6]; the use of these medicines in VIPs has been widely publicized [7]. Governments have also been spending a lot of time and effort in procuring and distributing remdesivir. Better action would have been disseminating evidence-based guidelines to ensure rational use of medications.

Initially, due to possibility of aerosol generation and possible increase in the risk of transmission of infection to $\mathrm{HCW}$, heated humidified high-flow nasal cannula (HHHFNC) was not recommended and endotracheal intubation/noninvasive ventilation (NIV) was preferred in hypoxic patients. However, over a period of time, as experts suggested use of HHHFNC, its use became more frequent and this modality might have saved a huge number of lives. However, in the current scenario of shortage of oxygen, there are concerns about high oxygen requirements in this modality. The sudden and sharp rise in sick COVID-19 cases has led to a huge increase in demand for oxygen, which the current supplies are unable to meet. Diverting the oxygen used in industry, provided it is pure, may provide some relief. Simultaneously, health facilities need to proactively work toward more efficient use of this scarce resource. As the authorities have now become busy in arranging oxygen and other life-saving equipment, adequate measures to reduce transmission of infection are likely to get compromised.

After witnessing the devastating effect of second surge, we have learnt that COVID-19 is likely to have repeated surges, so we should not lower our guard. The extremely fragile and inadequate health infrastructure needs a huge boost in all aspects: there have to be short-term as well as long-term goals. After first surge of the pandemic, some attention was given to improve healthcare infrastructure, but still, it is far from the needs of the country with previously suboptimal health infrastructure and there is a pressing need for taking appropriate action to improve it. We should anticipate surges and strengthen infrastructure for a third surge or other pandemics. Instead of knee-jerk reaction of increasing beds, buying ventilators, etc., it is important to systematically develop infrastructure for healthcare including development of different levels of hospitals together with expansion of intensive care and have trained healthcare workers at all levels, who can handle epidemics other than COVID-19 also. Developing effective system for continuous surveillance and smart forecasting is the need of the hour. A key to success is to accurately collect information and interpret it correctly to contain the spread of infection early, instead of the usual denial responses. It is also important 
that as a country, we understand the importance of health and increase public spending on health infrastructure. We have to move toward being proactive rather than being reactive!

\section{Declarations}

\section{Conflict of Interest None.}

\section{References}

1. India's Covid 'storm': how did situation get so bad during second wave. Available at http://timesofindia.indiatimes.com/articleshow/ 82198023.cms?utm_source $=$ contentofinterest\&utm_medium $=$ text\&utm campaign=cppst. Accessed on 24 Apr 2021

2. National Centre for Disease Control. Graphical illustration of data from COVID-19 cases in India. Available at: https://ncdc.gov.in/ dashboard.php. Accessed on 24 Apr 2021

3. Park YJ, Farooq J, Cho J, et al. Fighting the war against COVID-19 via cell-based regenerative medicine: lessons learned from 1918
Spanish flu and other previous pandemics. Stem Cell Rev Rep. 2021;17:9-32.

4. Jain P. Covid-19 pandemic nearing endemic phase in Delhi, says Health Minister Satyendar Jain. India Today. 7 March 2021. Available at: https://www.indiatoday.in/coronavirus-outbreak/story/ delhi-covid-19-pandemic-endemic-phase-health-minister-satyendarjain-1776528-2021-03-07. Accessed on 24 Apr 2021

5. World Health Organization. Therapeutics and COVID-19: living guidelines 31st March 2021. WHO reference number: WHO/2019nCoV/therapeutics/2021.1. Available at: https:/www.who.int/ publications/i/item/WHO-2019-nCoV-therapeutics-2021.1. Accessed on 24 Apr 2021

6. NIH COVID-19 Treatment Guidelines. Therapeutic management of adults with COVID-19 Last Updated: April 21, 2021 Available at: https://www.covid19treatmentguidelines.nih.gov/therapeuticmanagement/. Accessed on 24 Apr 2021

7. Covid-hit Donald Trump treated with remdesivir: White House. Mint. 3 October 2020. Available at: https://www.livemint.com/ news/world/covid-hit-donald-trump-being-treated-with-remdesivirwhite-house-11601715590627.html. Accessed on 24 Apr 2021

Publisher's Note Springer Nature remains neutral with regard to jurisdictional claims in published maps and institutional affiliations. 